\title{
Apoio Social e Reganho de Peso Pós-Cirurgia Bariátrica: Estudo de Caso sobre Intervenção com Cuidador
}

\author{
Marcela Abreu-Rodrigues \\ Eliane Maria Fleury Seidl ${ }^{1}$ \\ Instituto de Psicologia da Universidade de Brasília, Brasília, DF, Brasil
}

\begin{abstract}
Resumo
O estudo de caso teve por objetivos: aplicar uma intervenção comportamental para adequação do apoio social em cuidador de paciente com reganho de peso pós-cirurgia bariátrica; verificar os efeitos da intervenção mediante a avaliação da percepção do paciente sobre a disponibilidade e satisfação com o apoio social e do peso corporal, antes (linha de base), imediatamente após (momento 2) e um mês depois da intervenção (momento 3). A paciente tinha 20 anos e apresentava reganho de peso da ordem de $30 \%$ do peso mínimo alcançado após a cirurgia. A cuidadora era sua mãe, 43 anos, com quem residia. Entrevistas semiestruturadas para o cuidador e paciente e uma escala validada para avaliar o suporte social foram utilizados na coleta de dados. As intervenções com o cuidador consistiram de cinco sessões semanais nas quais técnicas de reforçamento diferencial, automonitoramento das condutas de apoio social e resolução de problemas foram utilizadas. Constatou-se aumento na frequência de fornecimento de apoio social por parte do cuidador nas quatro categorias comportamentais trabalhadas: adesão à dieta, uso de suplementação alimentar, prática de atividade física e controle de estresse, ao longo de três semanas da intervenção. Os resultados mostraram que, após a intervenção, houve um aumento dos escores da escala respondida pela paciente, se comparados ao da linha de base, bem como a redução do peso corporal ( $\mathrm{LB}=84 \mathrm{~kg}$; momento $3=82,2 \mathrm{~kg}$ ). $O$ estudo indica a possibilidade de aplicação de técnicas comportamentais para melhorar o apoio social de cuidadores de pacientes com reganho de peso pós-cirurgia bariátrica.
\end{abstract}

Palavras-chave: Apoio social, cuidador, reganho de peso, cirurgia bariátrica, intervenção comportamental.

\section{Social Support and Weight Regain After Bariatric Surgery: A Case Study of an Intervention with a Caregiver}

\begin{abstract}
The case study aimed to: implement a behavioral intervention for adequacy of social support in caregivers of patients with weight regain after bariatric surgery; verify the effects of the intervention by assessing the patient's perception of the availability and satisfaction with social support and body weight before (baseline), immediately after (moment 2) and one month after the intervention (moment 3 [M3]). The patient was a 20 -year-old woman, having a $30 \%$ weight regain in relation to the minimum weight achieved after surgery. The caregiver was her mother a 43 year old with whom he resided. Semi structured interviews for the caregiver and patient and a validated scale to access social support were

Endereço para correspondência: Universidade de Brasília, Instituto de Psicologia, Campus Universitário Darcy Ribeiro, Brasília, DF, Brasil 70910-900. E-mail: marcelabreu81@gmail.com

$\mathrm{O}$ artigo é parte da tese de doutorado da primeira autora, com apoio financeiro da Coordenação de Aperfeiçoamento de Pessoal de Nível Superior (CAPES), sob orientação da segunda autora.
\end{abstract}


used for data collection. Interventions with the caregiver consisted of five weekly sessions including differential reinforcement techniques, self-monitoring of social support and problem-solving. Following the two moments, there was an increase in the frequency of social support provision by the caregiver in the four behavioral categories worked in intervention: adherence to diet, use of supplements, physical activity and stress management, along three weeks. The results showed that after the intervention, there was an increase in scores on the Social Support Scale answered by the patient compared to the baseline and weight reduction $(\mathrm{LB}=185,1 \mathrm{lb} ; \mathrm{M} 3=180,7 \mathrm{lb})$. The study indicates the possibility of application of behavioral techniques to improve social support for caregivers of patient's regained weight after bariatric surgery.

Keywords: Social support, caregiver, weight regain, bariatric surgery, behavioral intervention.

\section{El Apoyo Social y la Recuperación del Peso Después de la Cirugía Bariátrica: Un Estudio de Caso de una Intervención con un Cuidador}

\section{Resumen}

El estudio de caso tuvo como objetivos: implementar una intervención conductual para la adecuación del apoyo social en cuidador de paciente con peso recuperado después de la cirugía bariátrica; investigar los efectos de la intervención mediante la evaluación de la percepción del paciente sobre la disponibilidad y la satisfacción con el apoyo social y el peso corporal, antes, inmediatamente después y un mes después de la intervención. El paciente tenía 20 años, estudiante universitario, había recuperado de peso en el orden de $30 \%$ del peso mínimo alcanzado después de la cirugía. El cuidador era su madre, 43 años, con quien residía. Entrevistas semiestructuradas para el cuidador y el paciente y uma escala validada para evaluar el apoyo social fueron utilizados para la recolección de datos. Las intervenciones con el cuidador consistieron en cinco sesiones en las que las técnicas de refuerzo, automonitoreo de los comportamientos sociales de apoyo y resolución de problemas se utilizaron. Durante la intervención se observó un aumento en la frecuencia de apoyo social del cuidador en las cuatro categorías de comportamento trabajadas: la adherencia a la dieta, el uso de suplementos, la actividad física y manejo del estrés. Los resultados mostraron que, después de la intervención, hubo un aumento en las puntuaciones de escala respondidas por el paciente, en comparación con la línea de base y la reducción de peso ( $\mathrm{LB}=185,1 \mathrm{lb}$; tiempo $3[\mathrm{~T} 3]=180,7 \mathrm{lb}$ ). El estudio indica la posibilidad de aplicación de intervención conductual para mejorar el apoyo social de los cuidadores de pacientes bariatricos con recuperaron del peso.

Palabras clave: Apoyo social, cuidador, peso recuperado, cirugía bariátrica, intervención conductual.

A obesidade é epidêmica em vários países, considerada pela Organização Mundial de Saúde um dos dez principais problemas de saúde pública do mundo, já que $12 \%$ da população mundial é obesa (World Health Organization [WHO], 2011). No Brasil, neste mesmo período, $14,8 \%$ da população adulta brasileira estava obesa, com prevalência mais elevada na faixa etária entre 55 e 64 anos $(21,3 \%)$ e entre mulheres (16,9\%). Dados de 2010 mostraram um recente aumento, sendo $15,8 \%$ da população brasileira portadora de obesidade $-15,6 \%$ dos homens e $16 \%$ das mulheres brasileiras (Ministério da Saúde, 2011).

Por ser considerada uma condição médica crônica de etiologia multifatorial, o seu tratamento envolve vários tipos de abordagens (Segal $\&$ Fandiño, 2002). Nessa perspectiva, intervenções como a prescrição de dietas de baixa caloria, programas de exercícios físicos, tratamento farmacológico e acompanhamento psicológico tendem a favorecer a perda de peso (Fichter \& Leibbrand, 2002). Essa realidade coloca de forma contundente a necessidade de uma abor- 
dagem interdisciplinar na atenção e cuidado a pacientes obesos, valorizando aspectos físicos, psicológicos e sociais das pessoas acometidas. Entretanto, a eficácia de tais intervenções tem se mostrado limitada no que se refere à manutenção da perda de peso em longo prazo em pacientes com obesidade severa (Ades \& Kerbauy, 2002), fato que justificou o aparecimento da cirurgia bariátrica como a tecnologia padrão ouro no tratamento da obesidade mórbida, nos últimos 30 anos. Assim, nesse cenário, profissionais de saúde passaram a buscar - não somente a sobrevivência de pacientes egressos dessa modalidade de cirurgia - mas a melhora de sua qualidade de vida e a manutenção do peso adequado.

\section{A Cirurgia Bariátrica}

O tratamento cirúrgico da obesidade mórbida é uma alternativa eficaz, embora radical, a ser empregada quando falham os métodos clínicos tradicionais. Segundo Benedetti (2009), a necessidade da cirurgia impõe-se pelo fato de a maioria dos obesos mórbidos não terem resultados satisfatórios quando se utilizam de tratamentos clínicos para emagrecer. Vale ressaltar que a cirurgia bariátrica sido empregada no tratamento de pessoas com IMC (índice de massa corporal) entre 30 e $35 \mathrm{~kg} / \mathrm{m}^{2}$ com ocorrência de doenças associadas, obrigatoriamente classificadas como graves por médico especialista na respectiva área da doença, e por constatação, também obrigatória, de "intratabilidade clínica da obesidade" por médico endocrinologista (WHO, 2011).

Ressalta-se que a qualidade técnica da cirurgia não garante nem emagrecimento nem melhor qualidade de vida. $\mathrm{O}$ emagrecimento não é consequência direta da intervenção cirúrgica bem sucedida, pois o fator determinante para o sucesso do tratamento é a forma como o paciente vai lidar com o novo contexto alimentar e de tratamento associado à redução do estômago (Benedetti, 2009). A cirurgia representa apenas uma etapa do processo. É necessário que haja, por parte dos pacientes operados, o compromisso de assumir uma mudança de estilo de vida, principalmente no que se refere à qualidade e quantidade da alimentação ingerida (Franques \& Areneles-Loli, 2006; Marchesini, 2010). Por essa razão, sem uma mudança radical no comportamento alimentar, a cirurgia perde o potencial emagrecedor gerando frustração em uma parcela dos pacientes que não consegue alcançar a redução esperada do seu peso corporal.

Segundo Benedetti (2009), o trabalho do psicólogo na cirurgia bariátrica não se restringe a submeter os pacientes a avaliações para diferenciar aqueles que podem dos que ainda não estão preparados para serem operados, mas acompanhá-los em sua trajetória, oferecendo-lhes preparação psicológica para que alcancem suas expectativas e objetivos, assim como os do seu médico e da equipe. Assim, a preparação psicológica tornou-se um procedimento padrão em várias equipes de saúde no Brasil e no mundo. Tais programas trabalham no sentido de redimensionar as expectativas do paciente, assim com incentivar uma mudança no estilo de vida antes da cirurgia. Contudo, a fase pós-cirúrgica tem se constituído em grandes desafios devido ao reganho de peso.

\section{Reganho de Peso Pós-Cirurgia Bariátrica}

Pacientes que se submetem à cirurgia bariátrica depositam no procedimento a "cura da obesidade" e falsamente acreditam que a cirurgia irá trazer as mudanças comportamentais necessárias para manter a perda de peso. A cirurgia bariátrica não é garantia de sucesso e vários cuidados pós-operatórios relevantes são requeridos. Assim, nem todos os pacientes submetidos ao procedimento cirúrgico desfrutam de uma perda de peso acentuada. Ademais, existe um número elevado de pacientes que recuperam uma grande parte do peso perdido (Bastos, Barbosa, Soriano, Santos, \& Vasconcelos, 2013; Eisenberg, Duffy, \& Bell, 2010). Espera-se que entre 20 a $25 \%$ do peso perdido será recuperado em até 10 anos, sendo que o reganho ocorre entre sete a $50 \%$ dos casos (Heber et al., 2010; Herpertz, Kielmann, Wolf, Haberbrand, \& Senf, 2004). De acordo com esses autores, o reganho de peso está diretamente relacionado com a não adesão à dieta e não modificação de estilo de vida em longo prazo.

Para conquistar e manter a perda de peso após a cirurgia bariátrica, a adesão a um novo 
estilo de vida é crucial. A adesão inclui a adoção de uma dieta balanceada, uso de suplementação alimentar, prática de atividade física regular e manejo do estresse. Para isso, estratégias comportamentais e cognitivas são relevantes como estabelecimento de metas realistas, estratégias de controle de estímulos e modificação de crenças sobre alimentação (Odom et al., 2010). Esses autores realizaram um estudo com o objetivo de identificar os preditores de reganho de peso em pacientes submetidos à técnica cirúrgica denominada Bypass em y de Roux. Para isso, foi enviado a 203 pacientes submetidos a cirurgia bariátrica há mais de um ano um instrumento de autorrelato, desenvolvido para o estudo, com respostas dadas em escala Likert para investigar o reganho de peso e variáveis associadas. Os resultados mostraram que $79 \%$ dos participantes relataram reganho de peso, sendo que $13 \%$ deles informaram um aumento de mais de $15 \%$ do total de peso perdido, o que é considerado reganho significativo. Como preditores da manutenção de peso, variáveis como controle da compulsão alimentar e engajamento em atividades de automonitoramento foram relevantes. Por outro lado, variáveis como falta de autocontrole alimentar, falta de apoio familiar e uso de álcool e outras drogas estiveram associadas ao reganho de peso. Assim, o estudo mostrou evidências acerca da relação entre preditores comportamentais e reganho de peso após a cirurgia bariátrica.

Alguns estudos ressaltaram a relação entre a presença de comportamentos alimentares não compatíveis com a cirurgia e o reganho de peso, indicando que episódios de perda de controle alimentar ou maior descontrole alimentar no pós-operatório foram associados à pior perda ponderal (Colles, Dixon, \& O'Brien, 2008) e reganho de peso (Kofman, Lent, \& Swencionis, 2010; Odom et al., 2010). Assim, a cirurgia bariátrica força o paciente a restringir a quantidade de alimentos ingeridos, mas não leva, necessariamente, à melhora qualitativa da ingesta alimentar. Faria, Kelly, Lins e Faria (2010) revelaram que diferentes padrões de comportamento podem afetar a perda de peso após a cirurgia bariátrica: o comportamento de "beliscar" é definido como o consumo entre as refeições de alimentos que apresentam um valor calórico superior a $150 \mathrm{kcal}$ por porção, incluindo ingestão excessiva de gorduras e açúcares; outro padrão é o do "comedor de doces" que são indivíduos que consomem doces ou líquidos com adição de açúcar que somam mais de $150 \mathrm{kcal}$ por porção três vezes ou mais vezes por semana. Tais comportamentos, de acordo com os autores, podem levar ao consumo energético excessivo, ocasionando, por sua vez, uma perda de peso insuficiente ou o reganho de peso. Nesse contexto, considera-se que o tratamento pós-operatório do paciente bariátrico deve envolver intervenções que incluem a adesão à alimentação saudável, aumento da prática de atividade física e ajustamento psicossocial às necessidades da cirurgia (Kalarchian \& Marcus, 2003).

\section{Apoio Social}

Segundo Cobb (1976), suporte social pode ser definido como a "informação que leva o indivíduo a acreditar que ele é cuidado, amado, estimado e que pertence a uma rede social com obrigações mútuas" (p. 300). Cohen, Underwood e Gottlieb (2000) descreveram o apoio social como um processo que envolve interações com outras pessoas e que facilita o enfrentamento do estresse e outros estímulos aversivos. Em um estudo de revisão, na mesma linha de raciocínio, Uchino, Cacioppo e Kiecolt-Glaser (1996) encontraram evidências que as relações de apoio entre as pessoas as protegem contra riscos à saúde e de eventos estressantes da vida. Os autores afirmaram que a disponibilidade do suporte social pode, em contextos de adoecimento crônico, acelerar processos de recuperação e facilitar a adesão a tratamentos medicamentosos. Abreu-Rodrigues e Seidl (2008), em um estudo de revisão da literatura, mostraram que há evidências da relação positiva entre a disponibilidade de apoio social e melhora da saúde do paciente coronariano. Assim, as autoras afirmaram que a presença de suporte social prevê uma melhor adesão a comportamentos de saúde (prática de atividade física, alimentação saudável, redução de estresse), promovendo, assim, a melhora da saúde e da qualidade de vida desses pacientes. 
Com relação ao apoio social no contexto de cirurgia bariátrica, Livhits et al. (2011) realizaram um estudo de revisão de literatura que objetivou determinar os efeitos de grupos de apoio com pacientes ou de outras formas de apoio social no pós-operatório de cirurgia bariátrica. Os autores encontraram 934 artigos que relacionavam cirurgia bariátrica e suas consequências e perda de peso, sendo que desses somente dez estudos apontavam para a relação entre redução do peso corporal e apoio social. Desses dez estudos identificados, metade era sobre os efeitos de grupos de apoio (nos quais o paciente participava) na perda de peso pós-operatória; a outra metade relatava o papel de outras fontes de apoio (percepção do apoio da família e número de pessoas na rede social) e sua relação com perda de peso. Todos os estudos sobre grupos de apoio encontraram uma relação positiva significativa $(p \leq 0,05)$ entre a participação do paciente no grupo e perda de peso. Os estudos sobre a relação entre outras fontes de apoio e perda de peso demonstraram uma tendência positiva, porém, estatisticamente não significativa.

Partindo do pressuposto que o apoio social compatível com as necessidades do paciente favorece a mudança do comportamento alimentar para a redução de peso e a adesão do paciente obeso ao tratamento proposto, o presente estudo teve por objetivo implementar procedimento de intervenção e investigar seus efeitos para o desenvolvimento do apoio social de um cuidador de paciente submetida a cirurgia bariátrica com situação de reganho de peso, com vistas à adesão ao tratamento e perda do excesso de peso.

\section{Método}

Trata-se de estudo de caso, com avaliação antes (linha de base), logo após o término da intervenção (momento 2) e trinta dias depois da intervenção (momento 3 ).

\section{Descrição da Paciente e da Cuidadora}

O estudo contou com a participação de uma jovem de 20 anos, solteira, estudante universitária, com diagnóstico de reganho de peso após cirurgia bariátrica. A paciente vinha sendo acom- panhada em uma clínica particular de Brasília, especializada em tratamento e acompanhamento de pacientes obesos candidatos à cirurgia bariátrica onde a primeira autora atuava como psicóloga há cerca de seis anos. Dados da entrevista com a jovem revelaram que ela teve um reganho de peso de $30 \%$ a partir do peso mínimo alcançado após o procedimento cirúrgico, sendo que a cirurgia bariátrica tinha sido realizada há 24 meses. Na avaliação de linha de base do presente estudo a paciente apresentou peso igual a 81,2 $\mathrm{kg}$ sendo que seu peso mínimo, pós-cirurgia bariátrica, foi de $56,8 \mathrm{~kg}$.

Ela residia com a mãe, o pai e o avô materno. A pessoa indicada pela paciente foi a sua mãe para participar da intervenção na qualidade de cuidadora. A cuidadora tinha 48 anos, com ensino superior completo, funcionária pública $\mathrm{e}$ sempre residiu com a paciente. Mostrou-se receptiva e disposta a participar das etapas da intervenção, apresentando-se comunicativa e interessada.

\section{Instrumentos e Materiais}

Roteiro de Entrevista Semiestruturado para a Paciente. A entrevista com 22 questões (fechadas e abertas), elaborada para a pesquisa, teve por objetivo investigar dados sociodemográficos, histórico de reganho de peso pós-cirurgia bariátrica, adesão ao tratamento (alimentação e suplementação), prática de atividade física, motivação para a mudança, autoavaliação do bem-estar geral e indicação do cuidador para a participação na pesquisa.

Roteiro de Entrevista Semiestruturado para a Cuidadora. A entrevista, elaborada para a pesquisa, foi aplicada na linha de base e buscou obter dados sociodemográficos do cuidador, grau de parentesco/vínculo com o paciente, nível de percepção e preocupação com o reganho de peso do paciente, comportamentos que demonstravam tal preocupação, avaliação da adesão ao tratamento do paciente e disponibilidade para participar do estudo.

Escala de Suporte Social. A escala teve por objetivo avaliar a disponibilidade percebida e a satisfação com o apoio social emocional e instrumental, sendo respondida pela paciente. Foi utili- 
zado o instrumento validado por Seidl e Tróccoli (2006) denominado Escala de Suporte Social para Pessoas com HIV/Aids, que foi adaptada da Social Support Inventory for People who are Positive or Have Aids (Renwick, Halpen, Rudman, \& Friedland, 1999). A escolha dessa escala para o presente estudo deveu-se à adequação de seus itens para análise do apoio social em contextos de saúde e doença, independente da enfermidade que esteja acometendo o paciente. Outro motivo é que não foram identificados instrumentos específicos direcionados à mensuração do apoio social de pessoas com obesidade. Ademais, a escala foi utilizada em pesquisas anteriores, aplicada a pessoas portadoras de outros agravos, como lesão medular traumática (Ribeiro, 2005) e pacientes cardíacos (Abreu-Rodrigues \& Seidl, 2013; Moraes \& Dantas, 2007). São mensuradas duas categorias de suporte, totalizando 22 itens:

1. Suporte social instrumental (10 itens, alpha de Cronbach igual a 0,84 ) - percepção da satisfação quanto à disponibilidade de apoio no manejo ou resolução de questões operacionais do tratamento ou do cuidado de saúde, de atividades práticas do cotidiano, de ajuda material e/ou financeira;

2. Suporte social emocional (12 itens, alpha de Cronbach igual a 0,92) - percepção e satisfação quanto à disponibilidade de escuta, atenção, informação, estima, companhia e apoio emocional. As respostas foram dadas em escala Likert de cinco pontos para frequência do apoio ( $1=$ nunca; $5=$ sempre) e satisfação ( $1=$ muito insatisfeito; $5=$ muito satisfeito). Pode ser usada na versão unifatorial (escore geral) ou bifatorial (suporte emocional e suporte instrumental). No presente estudo foram feitas análises dos dois fatores independentes e do escore geral. Foi feita mudança de redação para a adaptação de alguns itens da escala no intuito de focalizar a questão da obesidade, mas sem descaracterizar seu conteúdo em relação à escala original. Os escores, variando de 1 a 5 , foram obtidos mediante média aritmética. Escores mais elevados indicaram maior disponibilidade percebida e/ou satisfação com o suporte social recebido.
Avaliação do Peso. Em uma balança convencional avaliou-se o peso corporal do paciente, em kilogramas, em três momentos distintos: linha de base, momento 2 e momento 3 . O paciente foi pesado na mesma balança nas três avaliações.

Folheto Educativo. Foi elaborado para o estudo pela pesquisadora, com características didáticas, contendo figuras e linguagem acessível, usado na intervenção com o cuidador sobre o conceito de suporte social e as maneiras como o mesmo pode ser disponibilizado ao paciente bariátrico. $\mathrm{O}$ folheto contém informações sobre a definição de suporte social compatível com as necessidades do paciente bariátrico para evitar reganho de peso: adesão à dieta alimentar, adesão à suplementação, prática de atividade física e controle do estresse (Heber et al., 2010).

Ficha de Automonitoramento (checklist) de Comportamentos de Apoio Social. Consiste de um checklist sobre o fornecimento ou não fornecimento de apoio social, por um período de sete dias. As cinco categorias comportamentais presentes no folheto educativo - adesão a uma dieta balanceada e à suplementação, adesão à prática de atividade física e manejo do estresse -, eram mencionadas e foi feita uma breve descrição das formas de fornecer suporte social para cada categoria, com base nas dificuldades do cuidador. Caso este tivesse emitido comportamentos compatíveis com o suporte social naquela categoria, ele faria o registro da ocorrência, ou seja, marcaria um $\mathrm{X}$ para aquele dia específico.

\section{Procedimentos}

Inicialmente, o estudo foi submetido e aprovado pelo Comitê de Ética em Pesquisa da Faculdade de Ciências da Saúde da Universidade de Brasília (protocolo 147.535.135.000.030). A intervenção comportamental teve como objetivo auxiliar o cuidador a fornecer apoio social compatível com as necessidades do paciente com reganho de peso, segundo o projeto terapêutico elaborado para a paciente. A cuidadora, selecionada pela paciente, assim como a própria paciente, consentiram em participar do estudo, mediante assinatura de Termo de Consentimento Livre e Esclarecido (TCLE) específico. Foram utili- 
zados instrumentos e materiais distintos para a paciente e para a cuidadora, tal como explicitado anteriormente. Todas as sessões foram gravadas em áudio e transcritas.

Coleta de Dados. Linha de base - com a paciente. Os objetivos e as características da intervenção foram apresentados à paciente. Em um encontro de aproximadamente 90 minutos, foi feita uma sensibilização para fortalecer a participação no estudo e minimizar desistência. Logo após firmar o TCLE, a paciente respondeu à entrevista semiestruturada, conforme roteiro pré-estabelecido com o objetivo de obter dados sociodemográficos e clínicos, com foco no reganho de peso. Em seguida, a participante respondeu à escala de suporte social. O acompanhamento psicológico individual foi oferecido a paciente, se houvesse interesse, depois da fase de seguimento.

A intervenção com a cuidadora. Foi feito um contato inicial por telefone com a pessoa (apoio social) indicada pela paciente (sua mãe), quando foi convidada a participar da pesquisa. Nesse contato, foi agendada a primeira sessão na clínica onde o estudo foi realizado. Foram realizadas cinco sessões individuais, descritas a seguir. (a) $l^{a}$ sessão. Os objetivos e a relevância da intervenção foram apresentados à participante. Foi feita uma sensibilização para fortalecer sua participação no estudo e minimizar desistência. Em seguida, foi iniciada a entrevista semiestruturada com o objetivo de verificar expectativas e conhecimento da cirurgia, bem como os motivos que consideravam relevantes para o reganho de peso da paciente. O objetivo principal da sessão foi fazer um levantamento do repertório comportamental atual de apoio social e de condutas que poderiam ser adquiridas e modificadas para serem trabalhadas em encontros subsequentes. Além disso, buscou-se verificar a percepção da própria cuidadora quanto à quantidade e qualidade do apoio que vinha fornecendo, obtendo seu relato quanto aos tipos de apoio que já fornecia e a importância que atribuía aos mesmos. Foi questionado, ainda, como ela poderia prover apoio compatível com as necessidades do paciente e em qual aspecto tinha mais dificuldade. Ao final, foi estabelecido, em acordo mútuo, o dia e horário da segunda sessão. Ao final, foi fornecido o folheto educativo que nortearia os temas da sessão seguinte. A cuidadora levava o folheto para leitura como tarefa de casa. (b) $2^{a}$ sessão. Essa intervenção foi baseada nos dados obtidos na entrevista semiestruturada com a paciente e com a cuidadora na $1^{\mathrm{a}}$ sessão. Durante esse encontro foi realizada intervenção psicoeducativa com o objetivo de fornecer informações acerca da obesidade, cirurgia bariátrica e aspectos comportamentais do emagrecimento. Além do panfleto educativo, recursos audiovisuais (vídeo de animação sobre a cirurgia bariátrica) foram utilizados durante essa sessão para facilitar a compreensão dos conceitos e procedimentos abordados. Além disso, foram dadas orientações acerca do conceito de apoio social e como poderia fornecê-lo de forma compatível com as necessidades da paciente a fim de promover a perda de peso. Foram solicitados comportamentos e situações (compatíveis ou não com as necessidades da paciente) de apoio social que ocorreram durante a semana. A intervenção consistiu em levantamento de estratégias de solução de problemas relacionados ao não fornecimento de apoio social ou ao fornecimento de apoio inadequado. Tais estratégias foram levantadas junto à participante respeitando sua individualidade e o contexto no qual estava inserida. Reforçamento diferencial de comportamentos relevantes e adequados foi disponibilizado pela psicóloga de acordo com os relatos da cuidadora. Tal reforçamento foi fornecido por meio de elogios e/ou sorrisos imediatamente após relatos adequados de fornecimento de apoio social compatível com o que foi trabalhado na sessão anterior. Nessa sessão foram definidos os comportamentos de apoio a serem adotados pela cuidadora nas categorias: adesão à dieta, adesão à suplementação, adesão à prática de atividade física e controle do estresse. Ao final dessa sessão, foi feita instrução acerca do preenchimento e entregue à cuidadora a ficha de automonitoramento em formato de checklist com o objetivo de verificar se os comportamentos abordados durante a sessão foram emitidos ao longo da semana. Foi enfatizada a importância que esse registro fosse feito e trazido na sessão seguinte. (c) $3^{a}$ sessão. Essa 
intervenção foi baseada nos dados trazidos pela cuidadora na sua ficha de automonitoramento. A intervenção consistiu, assim como na $2^{\mathrm{a}}$ sessão, em levantamento de estratégias de solução de problemas relacionados ao não fornecimento de apoio social ou ao fornecimento de apoio inadequado. Tais estratégias foram levantadas junto à participante respeitando sua individualidade e o contexto em que estava inserida. Reforçamento diferencial de comportamentos relevantes e adequados foi disponibilizado pela profissional de acordo com os relatos da cuidadora. Ao final da sessão, a cuidadora foi orientada a preencher novamente a ficha de automonitoramento ao longo da semana. (d) $4^{a}$ sessão. Assim como na terceira sessão, a intervenção foi baseada na ficha de automonitoramento trazida pela cuidadora, sendo que estratégias de solução de problemas e reforçamento diferencial foram usadas. O conteúdo dessa sessão foi variável, voltado para as especificidades e demandas específicas do caso. (e) $5^{a}$ sessão. Intervenção semelhante à quarta sessão e obtenção de feedback acerca da intervenção de parte da cuidadora.

Seguimento. A paciente foi convidada a responder à Escala de Suporte Social e realizar a pesagem imediatamente após o término da intervenção (momento 2), assim como um mês após a intervenção específica (momento 3 ).

\section{Resultados}

\section{Linha de Base}

O reganho de peso da paciente iniciou aproximadamente um ano após a cirurgia, sendo que os motivos do mesmo, relatados pela paciente foram: beliscos frequentes, não prática de atividade física, ociosidade e ingestão de bebidas alcoólicas. A paciente expressou preocupação com o reganho de peso, conforme seu relato: "choro quase todos os dias quando me olho no espelho e vejo que estou engordando novamente. Te procurei porque quero ajuda para acabar com essa agonia, doutora".

$\mathrm{Na}$ entrevista feita com a cuidadora, esta relatou que a paciente não praticava atividade física, tinha uma adesão muito pobre à dieta prescri- ta e à suplementação e comparecia às consultas com o médico apenas uma vez ao ano.

No escore da Escala de Suporte Social, a paciente obteve um escore baixo $(1,6)$ na linha de base em termos de escore geral. No que se refere ao apoio instrumental, a paciente apresentou um escore de 1,6 e no apoio emocional o seu escore foi 1,7 . No instrumento, a participante assinalou a metade dos itens ( 11 itens) com valores 2 ou 3 na escala de respostas, indicando percepção de apoio insuficiente e insatisfatório. Ressalta-se que a paciente apresentou algumas respostas de não ocorrência (nunca), indícios de eventual inadequação do suporte social.

\section{Intervenção}

Primeira Sessão. A cuidadora, em todos os momentos da pesquisa, mostrou-se receptiva e disposta a participar, apresentando-se comunicativa e interessada. A cuidadora, ao ser questionada sobre quais comportamentos poderiam ser emitidos, compatíveis com as necessidades atuais do tratamento da filha, referiu aspectos emocionais, tal como exemplificado na fala:

Acho que devo ficar mais perto dela, ser mais carinhosa. Eu brigo muito com ela doutora, não gosto de vê-la gorda de novo! Ela já sofreu demais por ser gorda. Sofria bullying na escola, as primas gozavam dela, era um horror.

Verbalizou também:

Não sei doutora, eu não faço a comida dela. Como eu trabalho, ela come na rua ou na casa da minha mãe. Não quer ir mais lá na minha mãe pois diz a que a mesma fica falando que ela está gorda. Ela só gosta de ficar com os amigos dela, e ai ela bebe, doutora. Será que devo prender ela em casa? Esconder as comidas... é isso?

Tais relatos indicaram a necessidade de aprimorar condutas de apoio instrumental e modificar o apoio afetivo-emocional que parecia conter elementos aversivos e punitivos. Ademais, seus relatos indicavam que tinha dúvidas e estava insegura acerca do que fazer para ajudar a filha em seu processo de mudança. Os relatos da cuidadora indicaram ainda a ocorrência de um padrão 
ansioso diante da situação de reganho de peso da paciente que poderia afetar de forma negativa a relação mãe-filha.

Ao final da sessão, foi entregue o folheto informativo, feita a leitura do mesmo juntamente com a cuidadora e solicitada sua releitura em casa. Diante dessa tarefa, a cuidadora solicitou mais dois folhetos: um para entregar ao marido e outro para a mãe dela, avó materna da paciente. A pesquisadora entregou os folhetos à cuidadora e elogiou sua intenção no sentido de ampliar essas informações sobre apoio social para outros integrantes da família que conviviam com a paciente.

Sessão 2. Inicialmente, a cuidadora definiu suporte social, exemplificando com ações de natureza emocional e instrumental, especificando algumas formas de apoio tais como: "Levar a pessoa que precisa ao médico, cuidar dessa pessoa. Brigar com a pessoa doente para não fazer coisas erradas".

A cuidadora realizou a tarefa de leitura do folheto informativo e mostrou compreensão com relação às informações apresentadas. Ao ser questionada sobre as facilidades e dificuldades quanto a disponibilizar apoio à filha, a cuidadora relatou desafios na administração dos suplementos e na adesão à atividade física, exemplificados com as seguintes verbalizações:

Minha filha simplesmente abandonou o tratamento. Não toma mais nenhum suplemento. Ela disse que como não está fazendo atividade física, não precisa usar suplementos, isso é verdade, doutora? Ela disse que se tomasse sem malhar, iria engordar. Acreditei nela. Eu tirei ela da academia porque vi que estava pagando a toa. Ela falta mais do que vai, só quer saber de farra. Fica cansada nos outros dias e não vai à academia. Não achei meu dinheiro no lixo, né, doutora?

No que tange à alimentação, referiu a presença de algumas contingências que poderiam se constituir em risco para alimentação não compatível com as necessidades da cirurgia bariátrica:

Tem dias em que ela sai para a casa dos seus amigos ou vai comer na rua, lá eu não sei o que ela come. Ela diz que come pouco lá também, mas às vezes eu duvido. A M [paciente] adora uma besteira. Quando ela chega de madrugada da farra, ela faz miojo, ou come danoninho ou arroz com ovo. Ela não dorme bem e passa a noite toda comendo, fora a bebida que ingere nas festas, que eu sei.

Quanto às facilidades nesse aspecto relatou que "em geral, vejo que ela come em pouca quantidade, e gosta de comer tudo: frutas, verduras, integrais, leite, carnes e não é compulsiva como antes".

As dificuldades em fornecer suporte social à paciente relacionava-se também à categoria controle de estresse. Diante desse quadro, a cuidadora apontou a ociosidade e as brigas frequentes em casa com a filha como principal motivo de estresse:

Ela era super ativa assim que começou a emagrecer. Estava na faculdade, tinha namoradinhos, malhava, tinha planos. Mas agora nas férias, passa o dia sem fazer nada e fica comendo. A farra, a falta de trabalho e de atividade eu acho que é o maior problema dela. Fica nervosa, briga comigo e com o pai o tempo todo. Só quer saber de rua e de bebida com os amigos. Só não largou a faculdade, doutora, porque é lá que estão os amigos da farra. Diz que prefere os amigos do que ficar em casa.

Após apresentação do vídeo e da intervenção psicoeducativa, foram pactuadas as condutas prioritárias da cuidadora em relação às dificuldades da paciente, tais como: (a) comprar e verificar o uso da suplementação pela paciente e sensibilizá-la sobre a importância do uso contínuo da mesma: perguntar sobre ingestão de suplementos e se há necessidade de comprar quando perceber que está acabando; (b) participar da alimentação da paciente: observar se ingeriu alimentos adequados e reforçar essas condutas, verificar dieta prescrita junto à paciente, ter em casa alimentos compatíveis com a dieta prescrita, não comprar alimentos que possam contribuir para o reganho de peso da paciente (doces, carboidratos, por exemplo), incentivar consumo de alimentos adequados, reforçar essa conduta por meio de elogios, roupas novas, carinho, atenção; (c) verificar e apoiar a prática de atividade física: 
incentivar a prática de atividade física, negociar com a paciente a volta para a academia com uso de reforçamento positivo; (d) controle do estresse: propor e incentivar realização de atividades rotineiras, distrativas e prazerosas em família, para diminuir sua ociosidade e melhorar a comunicação com a paciente.

Foi feita a sensibilização e o treino de registro da ficha de automonitoramento junto à cuidadora que mostrou interesse e compreensão diante das informações apresentadas.

Sessões 3, 4 e 5. As informações da Figura 1 revelam que, no geral, houve melhora no fornecimento de apoio social da terceira sessão para a quinta sessão: houve aumento na frequência em todas as categorias exceto no que concerne à atividade física. Esse fato foi atribuído pela cuidadora, dentre outros aspectos, à virose contraída pela paciente no período da coleta de dados.

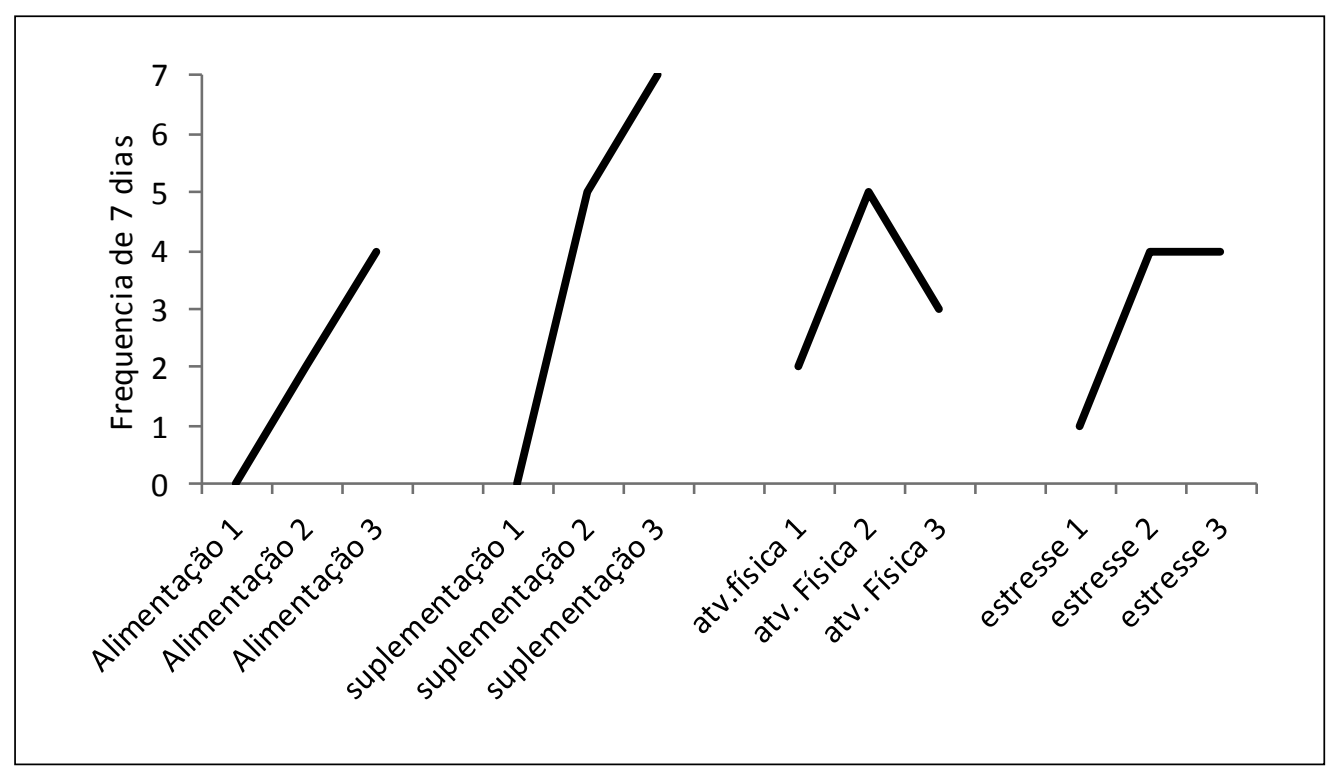

Figura 1. Respostas de apoio social da cuidadora nas categorias alimentação, suplementação, atividade física e controle do estresse da paciente, em um período de três semanas, medido pelo registro de automonitoramento.

Especificamente, em relação à alimentação, houve uma melhora de nenhum apoio social fornecido, para quatro apoios fornecidos na quinta sessão. Segundo a cuidadora, isso se deveu à melhor comunicação com a filha:

Depois que $M$ [a paciente] veio aqui doutora, ela me questionou sobre a nossa conversa. Expliquei que queria ajudá-la e que estava percebendo a sua infelicidade por estar engordando novamente. Nesse mesmo dia, eu a chamei para ir ao supermercado e compramos alimentos super saudáveis: frutas, verduras, integrais, peixe, frango. Acho que isso vai ser bom para a casa, estou gordinha também e meu pai é diabético. Outra coisa que aconteceu, é que passei a cozinhar e deixar comida em casa, pelo menos 3 vezes por semana. Faço o arroz e a carne sem gordura e dá para dois dias. M tem almoçado em casa e voltou para a minha mãe, lá é tudo bem saudável.

Outro comportamento em relação ao qual se observou aumento significativo da frequência (de zero para sete ocorrências) foi a adesão à suplementação. Inicialmente, a cuidadora não se preocupava com esse comportamento da paciente, não sabia acerca de sua importância. A intervenção focalizou na psicoeducação e nas formas de fornecimento de apoio. Depois das orientações fornecidas, houve uma maior participação da cuidadora e, consequentemente, um aumento na adesão da paciente, tal como relatou:

Expliquei para $M$ sobre a importância de se usar os suplementos e disse a ela que iríamos comprá-los. Negociei com ela, disse que se usasse os suplementos pagaria a 
academia que tanto quer frequentar, uma bem mais cara. Ela topou na hora [risos]. Todos os dias deixo a proteina pronta antes de ir trabalhar com os comprimidos do lado. Acho que ela toma, diz que toma, mas fiz minha parte.

Com relação ao controle de estressse, houve um aumento da terceira para a quarta sessão de uma para quatro respostas de apoio e a mesma se manteve na quinta sessão. Esse comportamento foi visto pela cuidadora como o mais prejudicial e com maior número de verbalizações e queixas da cuidadora. Ela expressou a esse respeito:

As brigas lá em casa com M. são constantes. Fico muito estressada quando ela sai com os amigos e volta de madrugada, doutora. Brigo muito com ela, e ela é super grosseira comigo. Além disso, brigo com ela por causa da gordura. Ela tá muito desleixada, sem vaidade nenhuma, não sei mais o que fazer. A rotina dela é praticamente ficar em casa o dia todo vendo TV e sair com os amigos pra beber ou comer à noite. Ela bebe todos os finais de semana.

Diante dessa situação, a intervenção focalizou em formas de fornecer suporte a fim de reduzir o nível de estresse da paciente. Tais procedimentos consistiram em estimular a paciente a fazer atividades cotidianas como pagar contas de casa, fazer a salada do almoço, levar o avô para a hemodiálise, consertar alguma coisa da casa, fazer compras (frutas, verduras) semanais, comprar pão, sendo necessário reforçar positivamente quando esta aderisse a tais atividades e aplicar técnica de extinção a eventuais comportamentos agressivos (verbais) da paciente. Com relação às saídas, foi pactuada que esta poderia negociar horários e aplicar reforço (social e material) no caso de cumprimento das regras, conversar com a paciente sobre o abuso de bebidas alcoólicas e sua relação com a cirurgia (risco de deficiência vitamínica e reganho de peso), bem como reforçar comportamentos não compatíveis com o uso abusivo de bebidas alcoólicas. Um relato exemplifica a melhora nas três fases da intervenção:

Se ocupou muito com a responsabilidade de levar o avô à hemodiálise. Passei a pagar 50 reais por semana a ela para levar o avô. Foi ótima ideia. As frutas e verduras ela não compra não, doutora. Mas se inscreveu no CCAA [Centro Cultural Anglo Americano] para estágio e levou o currículo em duas empresas. Reforcei ela levando ao shopping. Assim que voltou à academia, parou de beber. Saiu com as amigas para uma festa, mas chegou cedo e vi que não bebeu. Elogiei muito doutora, muito mesmo. Depois teve uma festa na casa da tia e ela bebeu que só. Não briguei muito porque estava na casa da tia dela, mas conversei no outro dia. Tem que ser aos poucos, né? Ela passou a ir para a academia quase que todos os dias, ela me buscava no trabalho e ia direto. Mas nessa última semana, eu tive que ficar no trabalho até mais tarde alguns dias e nem me preocupei em saber se ela estava frequentando ou não.

$\mathrm{Na}$ última sessão, ao ser questionada sobre sua opinião a respeito da intervenção, a cuidadora referiu melhora significativa no relacionamento com sua filha exemplificado no seguinte relato: "Eu e M estamos mais calmas. Vi que ela está empenhada e eu passei a ver mais coisas boas nela. Brigamos menos esses dias". Fez ainda uma avaliação positiva da intervenção, fazendo menção às mudanças na sua forma de fornecer apoio social à filha e aos ganhos observados, como por exemplo, sua adesão à dieta, à suplementação e à atividade física.

Momento 2 e Momento 3. Os escores totais da Escala de Suporte Social obtidos pela paciente após a intervenção com sua cuidadora passaram para 2,9 no momento 2 e, um mês após a intervenção, esse valor chegou a 3,3. Esses escores indicam uma melhora na disponibilidade e satisfação com apoio recebido na fase de seguimento. Além disso, houve uma redução de 1,8 $\mathrm{kg}$ no peso da paciente ao longo do processo: em LB a paciente apresentou o peso de $84 \mathrm{~kg}$, passando para $83,2 \mathrm{~kg}$ no momento 2 e $82,2 \mathrm{~kg}$ no momento 3 .

\section{Discussão}

Antes da intervenção os relatos da paciente indicaram a presença de insatisfações com a 
condição de reganho, dificuldades psicológicas, sociais e de adesão ao tratamento da participante, além de dificuldades de compreensão das reais necessidades do paciente bariátrico pela cuidadora. Outro desafio se referiu ao fato de que o tratamento para pessoas submetidas à cirurgia bariátrica é uma cadeia de comportamentos interdependentes que precisam ser aprendidos, tanto pelos pacientes quanto por seus cuidadores (Segal \& Fandiño, 2002).

No primeiro contato com a cuidadora, os dados da entrevista semiestruturada revelaram que ela tinha algum conhecimento sobre aspectos do apoio social, como a sua importância para a saúde da paciente, bem como de algumas características instrumentais e emocionais desse suporte. Havia, no entanto, limitações específicas na compreensão do que poderia ser realizado por ela, na medida em que destacou aspectos genéricos da noção de apoio social.

Os resultados obtidos na avaliação durante a linha de base indicaram a presença de lacunas específicas na disponibilidade e/ou satisfação com o apoio social, tanto no componente instrumental como no emocional. Esses dados sobre o repertório de entrada da cuidadora corroboram os achados de Boutin-Foster (2005) e de Lutfi, Torquati, Sekhar e Richards (2006) no sentido de que o suporte social disponibilizado muitas vezes não é compatível com as reais necessidades do paciente, podendo estar inadequado e gerando insatisfação. Assim, além do conhecimento sobre a doença que acomete a pessoa, o cuidador deve estar atento à história individual e à forma como cada paciente convive e se adapta à sua condição de saúde.

A intervenção, nesse estudo, propiciou escuta profissional qualificada às dificuldades apresentadas pela paciente, segundo a percepção do seu apoio social, permitindo identificar práticas que poderiam aumentar a vulnerabilidade da mesma. Por outro lado, o levantamento do padrão comportamental inicial da cuidadora foi fundamental para identificar as condutas com maior custo de resposta e, assim, estabelecer passos para a intervenção em direção aos comportamentos-alvo. Após ter sido exposta às condições de fornecimento de informações e in- tervenção comportamental, com base nas dificuldades relatadas e nas necessidades psicossociais da paciente, a cuidadora modificou o padrão de fornecimento de suporte social em quatro categorias comportamentais (hábitos alimentares, uso de suplementação, prática de atividade física e controle de estresse), que são priorizados na prevenção do reganho de peso em pacientes submetidos à cirurgia bariátrica (Heber et al., 2010; Sallet, Sallet, \& Dixon, 2007).

Por fim, outro dado que revela efeitos positivos da intervenção foi a perda de peso de da paciente, identificada nos momentos 2 e 3 . Com a melhora da disponibilidade e da satisfação com o apoio recebido, além do contato com o serviço de saúde que passou a fazer, a paciente melhorou sua adesão a comportamentos compatíveis com as necessidades da cirurgia bariátrica e de prevenção do reganho de peso. Esses dados corroboram resultados descritos na literatura que afirmam existir uma relação positiva entre as variáveis apoio social e adesão ao tratamento em doenças crônicas (Cohen et al., 2000; Uchino et al., 1996). Apesar de não ser regra entre pacientes obesos, a perda de peso em um período breve de intervenção, pareceu decorrer do fato de a paciente ter aumentado a frequência do comportamento de aderir às orientações médicas e nutricionais já durante a intervenção com o apoio social e manteve esse padrão um mês após o procedimento. No entanto, considerando o período muito breve de seguimento do presente estudo, esse resultado deve ser considerado com parcimônia, dadas as dificuldades de manutenção da perda de peso em casos de obesidade em espaços curtos de tempo (Fichter \& Laibbrand, 2002).

Nessa perspectiva, Marchesini (2010) afirma que, para se ter sucesso na fase pós operatória a longo prazo, deve haver um compromisso do paciente e de sua família no que tange às recomendações médicas e nutricionais, ou seja, o paciente deve ser ativo no seu tratamento.

Uma das contribuições do estudo foi a utilização de técnicas comportamentais de fácil manejo e aplicação. Assim, o uso dessas técnicas na fase de intervenção junto à cuidadora mostrou-se eficaz na medida em que fortaleceu as respostas de apoio compatível com as necessi- 
dades da paciente e, consequentemente, aumentou a disponibilidade e satisfação do suporte social recebido.

Os resultados apontaram, ainda, que intervenções comportamentais podem ajudar cuidadores a fornecer suporte social mais adequado a pacientes com reganho de peso pós-cirurgia bariátrica e, assim, promover melhora da saúde e da qualidade de vida dessa população. Como apontado, a obesidade é um alvo de convergência de esforços de profissionais da área de saúde, sendo uma das maiores preocupações da atualidade em termos de saúde pública. O aumento nas taxas de morbimortalidade dessa população, além da luta diária em obter melhora da qualidade de vida, torna o estudo e os cuidados com essa clientela necessários. Entretanto, de modo interdisciplinar, deve-se ter em mente que os cuidadores são grandes aliados nessa luta e que, ao promover suporte social compatível com suas necessidades, estaremos promovendo a saúde e a qualidade de vida dos pacientes.

\section{Referências}

Abreu-Rodrigues, M., \& Seidl, E. M. F. (2008). A importância do apoio social em pacientes coronarianos. Paidéia (Ribeirão Preto), 18(40), 279288. doi:10.1590/S0103-863X2008000200006

Abreu-Rodrigues, M., \& Seidl, E. M. F. (2013). Apoio social a pacientes coronarianos: Intervenção comportamental com um familiar. Estudos de Psicologia (Campinas), 30(4), 619-628. doi:10.1590/S0103-166X2013000400015

Ades, L., \& Kerbauy, R. R. (2002). Obesidade: Realidade e indagações. Psicologia USP, 13, 197 216.

Bastos, E. C. L., Barbosa, E. M. W. G., Soriano, G. M. S., Santos, E. A., \& Vasconcelos, S. M. L. (2013). Fatores determinantes do reganho ponderal no pós operatório de cirurgia bariátrica. ABCD. Arquivo Brasileiro de Cirurgia Digestiva, 26(Supl. 1), 26-32. doi:10.1590/S010267202013000600007

Benedetti, C. (2009). De obeso a magro: A trajetória psicológica. São Paulo, SP: Vetor

Boutin-Foster, C. (2005). Getting to the heart of social support: A qualitative analysis of the types of instrumental support that are most helpful in motivating cardiac risk factor modification. Heart \& Lung, 34(1), 22-29. doi:10.1016/j. hrtlng.2004.09.002

Cobb, S. (1976). Social support as a moderator of life stress. Psychosomatic Medicine, 38(5), 300-314. doi:10.1097/00006842-197609000-00003

Cohen, S., Underwood, L. G., \& Gottlieb, H. B. (2000). Social support measurement and intervention. New York: Oxford University Press.

Colles, S. L., Dixon, J. B., \& O’Brien, P. E. (2008). Grazing and loss of control related to eating: Two high risk factors following bariatric surgery. Obesity, 16(3), 615-622. doi:10.1038/ oby.2007.101

Eisenberg, D., Duffy, A. J., \& Bell, R. L. (2010). Does preoperative weight change predict postoperative weight loss after laparoscopic Rouxen Y Gastric Bypass in short term? Journal of Obesity, 10, 1-4. doi:10.1155/2010/907097

Faria, S. L., Kelly, E. O., Lins, R. D., \& Faria, O. P. (2010). Nutritional management of weight regain after bariatric surgery. Obesity Surgery, 20, 135-139. doi:10.1007/s11695-008-9610-z

Fichter, M. M., \& Leibbrand, R. (2002). Maintenance of weight loss after obesity treatment: Is continuous support necessary? Behavior Research and Therapy, 40(11), 1275-1289. doi:10.1016/ S0005-7967(01)00099-7

Franques, A. R. M., \& Arenales-Loli, M. S (2006). Contribuições da psicologia na cirurgia da obesidade. São Paulo, SP: Vetor.

Heber, D., Greenway, F. L., Kaplan, L. M., Livingstone, E., Salvador, J., \& Still, C. (2010). Endocrine and nutritional management of the postbariatric surgery patient: An endocrine society clinical practice guideline. Journal of Clinical Endocrinology and Metabolism, 95, 4823-4843. doi:10.1210/jc.2009-2128

Herpertz, S., Kielmann, R., Wolf, A. M., Haberbrand, J., \& Senf, W. (2004). Do psychosocial variables predict weight loss or mental health after obesity surgery? A systematic review. Obesity Research, 12(10), 1554-1569. doi:10.1038/oby.2004.195

Kalarchian, M. A., \& Marcus, M. D. (2003). Management of the bariatric surgery patient: Is there a role for the cognitive behavioral therapist? Cognitive and Behavioral Practice, 10, 112-119. doi:10.1016/S1077-7229(03)80019-6 
Kofman, M. D., Lent, M. R., \& Swencionis, C. (2010). Maladaptive eating patterns, quality of life, and weight outcomes following gastric bypass: Results of an internet survey. Obesity, 18(10), 1938-1943. doi:10.1038/oby.2010.27

Livhits, M., Mercado, C., Yermilov, I., Parikh, J. A., Dutson, E., Mehran, A. ...Gibbons, M. M. (2011). Is social support associated with greater weight loss after bariatric surgery? A systematic review. Obesity Reviews, 12, 142-148. doi:10.1111/j.1467-789X.2010.00720.x

Lutfi, R., Torquati, A., Sekhar, N., \& Richards, W. O. (2006). Predictors of success after laparoscopic gastric bypass: A multivariate analysis of socioeconomic factors. Surgical Endoscopy, 20, 864867.

Marchesini, J. B. (2010). Acompanhamento psicológico tardio em pacientes submetidos à cirurgia bariátrica. ABCD. Arquivos Brasileiros de $\mathrm{Ci}$ rurgia Digestiva, 23(2), 113-120. doi:10.1590/ S0102-67202010000200010

Ministério da Saúde. (2011). Vigilância de fatores de risco e proteção para doenças crônicas por inquérito telefônico. Brasília, DF: Autor.

Moraes, T. P. R., \& Dantas, R. A. S. (2007). Avaliação do suporte social entre pacientes cardíacos cirúrgicos: Subsídio para o planejamento da assistência de enfermagem. Revista Latino-Americana de Enfermagem, 15(2), 323-329. doi:10.1590/S0104-11692007000200020

Odom, J., Zalesin, K. C., Washington, T. L., Miller, W. W., Hakmeh, B., Zaremba, D. L., ...McCullough, P. A. (2010). Behavioral predictors of weight regain after bariatric surgery. Obesity Surgery, 20, 349-256. doi:10.1007/s11695-0099895-6

Quase metade da população brasileira está acima do peso. (n.d.). Portal da Saúde. Recuperado em http://portalsaude.saude.gov.br/index. $\mathrm{php} / \mathrm{profissional-e-gestor/vigilancia/links-}$ vigilancia?start $=10$
Renwick, R., Halpen, T., Rudman, D., \& Friedland, J. (1999). Description and validation of a measure of received support specific to HIV. Psychological Reports, 84(2), 663-673. doi:10.2466/ PR0.84.2.663-673

Ribeiro, P. C. H. Y. (2005). Suporte Social e qualidade de vida em pessoas portadoras de lesão medular traumática (Dissertação de mestrado, Universidade de Brasília, DF, Brasil).

Sallet, P. C., Sallet, J. A., \& Dixon, J. B. (2007). Eating behavior as a prognosis factor for weight loss after gastric bypass. Obesity Surgery, 17(4), 445-451. doi:10.1007/s11695-007-9077-3

Seidl, E. M. F., \& Tróccoli, B. T. (2006). Desenvolvimento de escala para avaliação do suporte social em pessoas vivendo com HIV/aids. Psicologia Teoria e Pesquisa, 22(3), 317-326. doi:10.1590/ S0102-37722006000300008

Segal, A., \& Fandiño, J. (2002). Indicações e contra-indicações para a realização das operações bariátricas. Revista Brasileira de Psiquiatria, 24(Supl. 3), 68-72. doi:10.1590/S151644462002000700015

Uchino, N. B., Cacioppo, T. J., \& Kiecolt-Glaser, K. J. (1996). The relationship between social support and physiological processes: A review with emphasis on underlying mechanisms and implications for health. Psychological Bulletin, 119(3), 488-531.

World Health Organization. (2011). Death and daily estimates for 2011 by cause for WHO members states. Retrieved from http://www.who.int/healthinfo/bod/en/index.html 